\title{
Aleatoriedade e variabilidade produtiva de feijão-de-vagem
}

\author{
Randomness and variability productive of bean pod
}

\section{Daniel Santos $^{\mathrm{I}}$ Fernando Machado Haesbaert ${ }^{\mathrm{I}}$ Alessandro Dal’Col Lúcio ${ }^{\mathrm{I}}{ }^{*}$ Sidinei José Lopes ${ }^{\mathrm{II}}$ Alberto Cargnelutti Filho II Vilson Benz $^{\mathrm{I}}$}

\section{RESUMO}

Com o objetivo de estudar a aleatoriedade e a variabilidade produtiva de feijão-de-vagem, foram realizados os seguintes experimentos em branco: 1) em estufa no outonoinverno; 2) em túnel no outono-inverno; 3) em ambiente não protegido no outono-inverno; 4) em túnel na primavera-verão; e 5) em ambiente não protegido na primavera-verão. A variável mensurada foi a fitomassa fresca de vagens. Foram realizados estudos por colheita e por agrupamentos de colheitas. Foram planejados diferentes tamanhos de parcela e, para cada um, foram realizados testes de homogeneidade de variâncias entre as fileiras de cultivo e entre as colheitas, e testes de aleatoriedade em cada fileira de cultivo. A não aleatoriedade e a variabilidade da produção de fitomassa fresca de vagens são maiores em condições meteorológicas adversas a cultura do feijão-devagem e diminuem com o aumento do tamanho de parcela. $O$ uso de parcelas constituídas por seis unidades básicas (12 plantas), em ambiente protegido ou não protegido, torna a produção de fitomassa fresca de vagens aleatória e proporciona menor variabilidade entre fileiras de cultivo e entre colheitas.

Palavras-chave: Phaseolus vulgaris L., tamanho de parcela, precisão experimental.

\section{ABSTRACT}

With the aim of studying the randomness and variability of productive bean pod, the following experiments were performed on white: 1) in greenhouse in autumn-winter, 2) in tunnel in autumn-winter, 3) in unprotected environment in autumn-winter, 4) in tunnel in spring-summer and 5) in unprotected environment in spring-summer. The variable measured was the fresh weight of pods. Studies were conducted by harvest and harvest groupings. Were planned different plot sizes, and for each, were conducted tests of homogeneity of variances between crop row and between harvests, and tests of randomness in each crop row. The non-randomness and variability in production of fresh pods are larger in adverse weather conditions the culture of the bean pod and decrease with increasing plot size. The use of plots consisting of six basic units (12 plants) in protected or unprotected makes the production of fresh pods random and provides lower variability between rows and between harvests.

Key words: Phaseolus vulgaris L., plot size, experimental precision.

\section{INTRODUÇÃO}

O feijão-de-vagem (Phaseolus vulgaris L.) é o principal representante das fabáceas na olericultura, diferindo do feijão comum por fornecer vagens para consumo in natura (FILGUEIRA, 2000). É uma boa alternativa para diversificar a produção das propriedades, com uso crescente em rotação de culturas ou no período de entressafra de culturas, como a do pimentão e tomate, o qual, além de aproveitar o sistema de tutoramento e a adubação residual, serve para interromper o ciclo de algumas doenças.

Devido à crescente importância dessa cultura, diversas pesquisas vêm sendo realizadas com ela tanto em ambiente protegido como não protegido, visando à identificação de cultivares e estratégias de cultivo que possibilitem aumento na produtividade $\mathrm{e}$ na sustentabilidade do seu cultivo (PEREIRA et al., 2003; ABREU et al., 2004; HELDWEIN et al., 2010).

'Programa de Pós-graduação em Agronomia, Centro de Ciências Rurais (CCR), Universidade Federal de Santa Maria (UFSM), Santa Maria, RS, Brasil.

"Departamento de Fitotecnia, CCR, UFSM, 97105-900, Santa Maria, RS, Brasil. E-mail: adlucio@smail.ufsm.br. *Autor para correspondência. 
Diante disso, é importante que sejam realizados trabalhos que possibilitem uma redução da variância residual e tornem mais precisos e confiáveis os resultados dessas pesquisas.

Vários são os fatores que podem reduzir a precisão dos resultados de uma pesquisa. Dentre eles, pode-se citar o erro experimental elevado e o não atendimento dos pressupostos do modelo matemático da análise de variância, necessários para a validação das análises estatísticas. Para as grandes culturas, já foram realizados alguns estudos, nos quais se testou o atendimento aos pressupostos do modelo matemático, porém, para as culturas olerícolas, são raros. Estudando transformações para dados com excesso de zero, COUTO et al. (2009) constataram que a transformação raiz quarta melhora o atendimento da normalidade das variáveis número e fitomassa fresca de frutos da abobrinha italiana. ZANARDO et al. (2010), trabalhando com alface, verificaram que não há atendimento das pressuposições de homogeneidade e/ou normalidade dos erros experimentais para algumas variáveis produtivas da cultura estudada.

Uma estratégia eficiente para redução do erro experimental é a determinação dos tamanhos de parcela e de amostra e do número de repetições (STORCK et al., 2006). Essas determinações são diretamente influenciadas pela variabilidade inerente ao experimento (STEEL et al., 1997). Em experimentos com culturas olerícolas, além da heterogeneidade do solo, algumas particularidades, tais como: a presença ou a ausência de frutos aptos a serem colhidos em determinada colheita; as múltiplas colheitas realizadas para algumas culturas; e o manejo cultural intensivo, são fontes de variabilidade (LÚCIO et al., 2008).

Os trabalhos realizados por LOPES et al. (1998), com tomate; LÚCIO etal. (2006), com pimentão; LÚCIO et al. (2008) e CARPES et al. (2010), com abóbora italiana; SANTOS et al. (2010), com alface mostram existir variabilidade entre as fileiras de cultivo e entre as épocas de colheita, independentemente da cultura olerícola avaliada. Dessa forma, é necessário verificar o comportamento desta variabilidade no sentido da linha de cultivo, bem como identificar estratégias para reduzi-la.

O objetivo deste trabalho foi estudar a aleatoriedade e a variabilidade produtiva de feijão-devagem em diferentes tamanhos de parcela, em experimentos em ambiente protegido e não protegido.

\section{MATERIAL E MÉTODOS}

Os experimentos foram realizados na área experimental do Departamento de Fitotecnia no Campus da Universidade Federal de Santa Maria, Santa Maria, RS, latitude: $29^{\circ} 42^{\prime} 23$ 'S, longitude: 53 43' $15^{\prime \prime}$ W e altitude: 95 metros. O solo da área experimental é classificado no
Sistema Brasileiro de Classificação de Solos (EMBRAPA, 2006) como Argissolo Vermelho Distrófico arênico.

Foram realizados experimentos em branco no outono-inverno, onde todos os experimentos foram realizados de 23/03/09 a 03/07/09, e na primavera-verão, onde todos os experimentos foram realizados de 09/10/ 09 a 06/01/10. Os experimentos foram os seguintes: 1 ) em estufa no outono-inverno; 2) em túnel no outonoinverno; 3) em ambiente não protegido no outonoinverno; 4) em túnel na primavera-verão; e 5) em ambiente não protegido na primavera-verão.

A estufa plástica possui estrutura metálica do tipo arco pampeano, pé direito de $2 \mathrm{~m}$ e $3,5 \mathrm{~m}$ na parte central, com $20 \mathrm{~m}$ de comprimento e $10 \mathrm{~m}$ de largura, orientada no sentido norte-sul. O túnel possui pé direito de $3 \mathrm{~m}, 20 \mathrm{~m}$ de comprimento e também orientação nortesul. A cobertura dos ambientes protegidos foi feita com filme de polietileno de baixa densidade (PEBD), com espessura de 150 micras e aditivo anti-UV. A área utilizada para o cultivo não protegido e o túnel, foi a mesma nas duas épocas.

A cultivar de feijão-de-vagem utilizada foi a 'Macarrão', de crescimento indeterminado. Em todos os experimentos, a semeadura foi realizada em camalhões com $0,20 \mathrm{~m}$ de altura e $0,40 \mathrm{~m}$ de largura, cobertos com faixas de mulching de filme opaco de PEBD de cor preta. Na estufa, foram cultivados seis camalhões (fileiras de cultivo) com 72plantas cada, no túnel e no ambiente não protegido foram três camalhões, com 84 plantas cada. O espaçamento foi de $0,2 \mathrm{~m}$ entre plantas e de $1 \mathrm{~m}$ entre fileiras.

Duplas de plantas vizinhas na fileira de cultivo constituiram as unidades básicas (UB). As colheitas das vagens de cada UB foram realizadas em: 23/05/09 (C1), 05/06/09 (C2), 19/06/09 (C3) e 03/07/09 (C4), no outono-inverno, e em 18/12/09 (C1), 29/12/09 (C2); e, em 06/01/10 (C3), na primavera-verão. Imediatamente após cada colheita, as vagens foram pesadas em balança digital com precisão de 0,01 gramas para determinação da fitomassa fresca das vagens, sendo esse, o caráter utilizado nas análises estatísticas.

A fitomassa fresca das vagens foi analisada em cada colheita e nos seguintes agrupamentos de colheitas: $\mathrm{C} 1+\mathrm{C} 2, \mathrm{C} 3+\mathrm{C} 4, \mathrm{C} 1+\mathrm{C} 2+\mathrm{C} 3+\mathrm{C} 4$, no outonoinverno, e $\mathrm{C} 1+\mathrm{C} 2, \mathrm{C} 2+\mathrm{C} 3$ e $\mathrm{C} 1+\mathrm{C} 2+\mathrm{C} 3$, na primaveraverão. Foram planejados tamanhos de parcela múltiplos do número de unidades básicas por fileira de cultivo, de modo a utilizar todo o ambiente de cultivo, dessa forma, foram planejadas parcelas com 1, 2, 3 e $6 \mathrm{UB}$, ambos no sentido da fileira de cultivo. Para cada um dos tamanhos de parcela testados, foi estimada uma variância por fileira de cultivo em cada colheita. Testouse a homogeneidade dessas variâncias entre as colheitas e entre as fileiras de cultivo, através do teste de Bartlett (STEEL et al., 1997), em nível de 5\% de probabilidade de erro. 
Para verificar se a produção de fitomassa fresca de vagens foi aleatória, aplicou-se o teste de sequência (BEAVER et al., 1974), em nível de 5\% de probabilidade de erro, para cada tamanho de parcela em cada fileira de cultivo por colheita e agrupamentos de colheitas. Quando o número de parcelas por fileira de cultivo foi maior do que 20, o teste foi realizado usando-se a aproximação a distribuição normal. Para tanto, calcularam-se os valores de $\mathrm{E}(\mathrm{c}) \mathrm{e} \mathrm{V}(\mathrm{c})$ pelas equações $\mathrm{E}(c)=1+(2 \cdot m \cdot n / m+n) \mathrm{e}$ $\mathrm{V}(c)=2 . m . n \cdot(2 . m \cdot n-m-n) /(m+n)^{2} .(m+n-1)$, em que: $\mathrm{m}$ é o número de valores superiores à mediana $\mathrm{e} n$ número de valores inferiores à mediana; para, em seguida, calcular $Z$ pela equação $Z=c-E(c) / \sqrt{V(c)}$, em que: c é o número de sequências observado. A sequência de dados foi considerada aleatória quando o valor, em módulo, de $\mathrm{z}$ calculado foi menor que o valor de $\mathrm{z}$ tabelado, e não aleatória quando maior ou igual a $\mathrm{z}$ tabelado.

Quando o número de parcelas por fileira de cultivo foi menor do que 20 , o teste foi realizado determinado-se o nível mínimo de significância (nms), que é igual ao dobro do menor valor entre $\alpha_{1}$ $\mathrm{P}\left(\mathrm{c} \leq \mathrm{c}_{1}\right)$ e $\alpha_{2} \mathrm{P}\left(\mathrm{c} \geq \mathrm{c}_{2}\right)$ (tabelados). A sequência foi considerada aleatória quando o $\mathrm{nms}$ foi maior do que $\alpha(5 \%)$, e não aleatória quando menor ou igual a $\alpha$.

As variáveis meteorológicas foram obtidas na Estação Climatológica Principal do $8^{\circ}$ Distrito de Meteorologia do Instituto Nacional de Meteorologia, localizada a $100 \mathrm{~m}$ da área experimental.

\section{RESULTADOS E DISCUSSÃO}

Nos experimentos que tiveram, durante sua realização, um maior número de dias com radiação global incidente ( $\mathrm{Rg}$ ) abaixo do limite trófico (LT) e/ou temperatura média do ar fora da faixa ideal do feijão-devagem (Figura 1), a proporção de casos de não aleatoriedade foi maior (Tabelas 1 e 2). O limite trófico de uma cultura é considerado como nível mínimo de $\mathrm{Rg}$

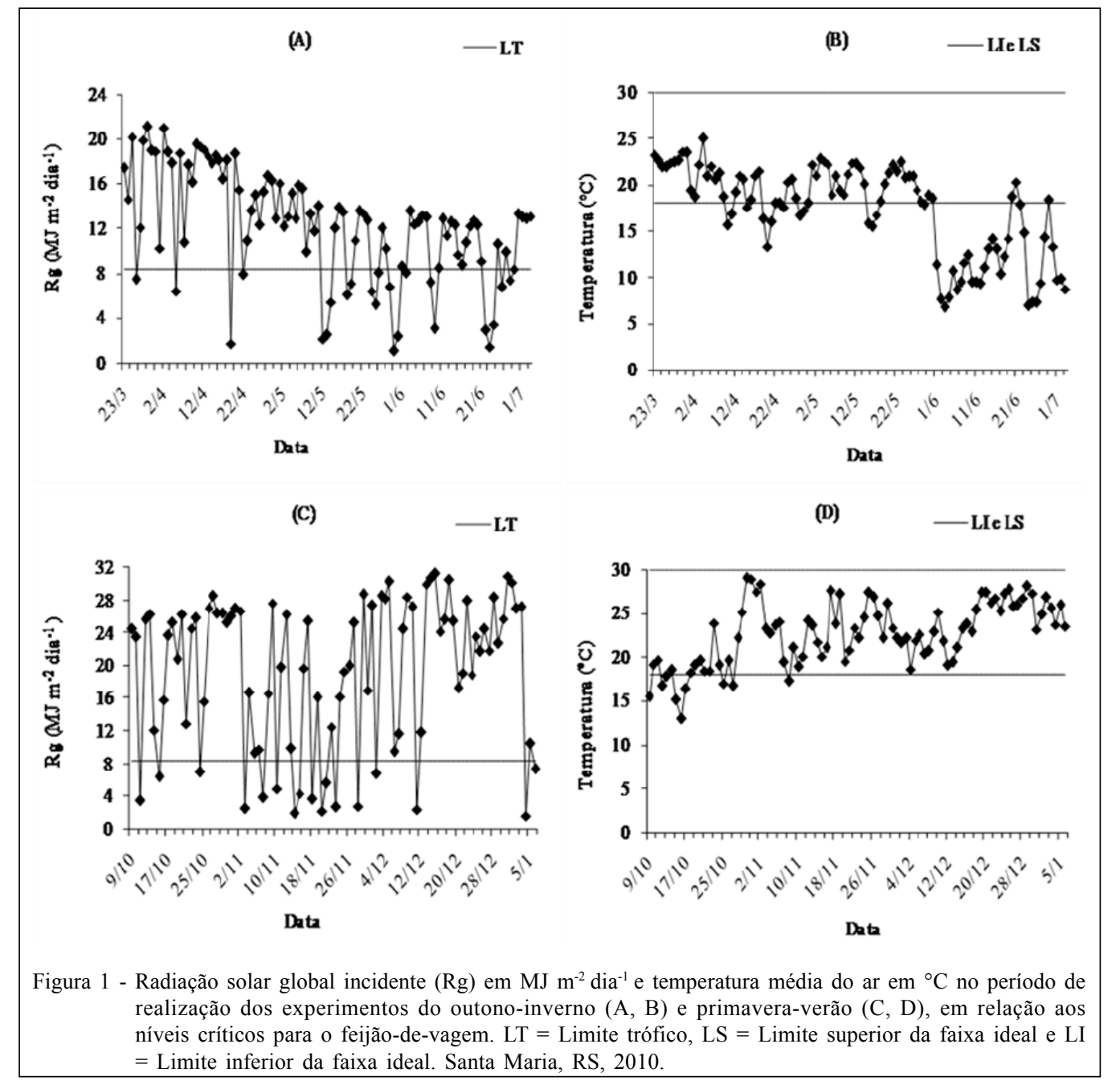

Ciência Rural, v.42, n.7, jul, 2012. 
Tabela 1 - Aleatoriedade da produção de fitomassa fresca de vagens para diferentes tamanhos de parcela, por fileira de cultivo, por colheita (C) e agrupamentos de colheitas de feijão-de-vagem (Phaseolus vulgaris L.) cultivado em estufa, túnel e em ambiente não protegido no outono-inverno. Santa Maria, RS, 2010.

\begin{tabular}{|c|c|c|c|c|c|c|c|c|c|c|c|c|c|}
\hline \multirow{3}{*}{$\mathrm{C}$} & \multirow{3}{*}{ Fileiras } & \multicolumn{12}{|c|}{ 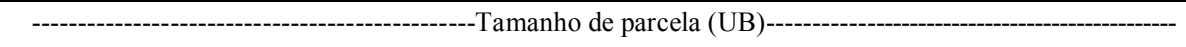 } \\
\hline & & 1 & 2 & 3 & 6 & 1 & 2 & 3 & 6 & 1 & 2 & 3 & 6 \\
\hline & & \multicolumn{8}{|c|}{-----------------Estufa------------------ } & \multicolumn{4}{|c|}{------------Não protegido----------- } \\
\hline \multirow{6}{*}{1} & 1 & A & A & A & A & A & A & A & A & A & $\mathrm{A}$ & NA & A \\
\hline & 2 & NA & A & A & A & A & A & A & A & NA & A & A & A \\
\hline & 3 & A & A & A & A & A & A & A & A & A & $\mathrm{A}$ & A & $\mathrm{A}$ \\
\hline & 4 & A & A & A & A & - & - & - & - & - & - & - & - \\
\hline & 5 & A & A & A & A & - & - & - & - & - & - & - & - \\
\hline & 6 & A & A & A & A & - & - & - & - & - & - & - & - \\
\hline \multirow{6}{*}{2} & 1 & NA & NA & A & A & A & A & A & A & NA & NA & NA & A \\
\hline & 2 & A & A & A & A & A & A & A & A & NA & NA & A & $\mathrm{A}$ \\
\hline & 3 & A & A & A & A & A & A & NA & A & A & A & A & $\mathrm{A}$ \\
\hline & 4 & A & A & A & A & - & - & - & - & - & - & - & - \\
\hline & 5 & NA & A & A & A & - & - & - & - & - & - & - & - \\
\hline & 6 & A & A & A & A & - & - & - & - & - & - & - & - \\
\hline \multirow{6}{*}{3} & 1 & A & A & A & A & A & A & A & A & NA & NA & NA & A \\
\hline & 2 & A & A & A & A & A & A & A & $\mathrm{A}$ & A & A & A & $\mathrm{A}$ \\
\hline & 3 & A & A & A & A & A & A & A & A & NA & A & A & A \\
\hline & 4 & A & A & A & A & - & - & - & - & - & - & - & - \\
\hline & 5 & A & A & A & A & - & - & - & - & - & - & - & - \\
\hline & 6 & A & A & A & A & - & - & - & - & - & - & - & - \\
\hline \multirow{6}{*}{4} & 1 & A & A & A & A & A & A & A & A & A & A & A & $\mathrm{A}$ \\
\hline & 2 & A & A & A & A & A & A & A & $\mathrm{A}$ & A & A & A & $\mathrm{A}$ \\
\hline & 3 & NA & A & A & A & A & A & A & A & A & A & A & $\mathrm{A}$ \\
\hline & 4 & A & A & A & A & - & - & - & - & - & - & - & - \\
\hline & 5 & A & A & A & A & - & - & - & - & - & - & - & - \\
\hline & 6 & A & A & A & A & - & - & - & - & - & - & - & - \\
\hline \multirow{6}{*}{$1+2$} & 1 & A & A & A & A & A & A & A & A & NA & NA & NA & A \\
\hline & 2 & NA & A & A & A & A & A & A & $\mathrm{A}$ & A & A & A & $\mathrm{A}$ \\
\hline & 3 & A & A & A & A & A & A & A & A & A & A & A & $\mathrm{A}$ \\
\hline & 4 & A & A & A & A & - & - & - & - & - & - & - & - \\
\hline & 5 & A & A & A & A & - & - & - & - & - & - & - & - \\
\hline & 6 & A & A & A & A & - & - & - & - & - & - & - & - \\
\hline \multirow{6}{*}{$3+4$} & 1 & A & A & A & A & A & A & A & $\mathrm{A}$ & A & NA & NA & $\mathrm{A}$ \\
\hline & 2 & A & A & A & A & A & A & A & A & A & A & A & $\mathrm{A}$ \\
\hline & 3 & A & A & A & A & A & A & A & A & A & A & A & A \\
\hline & 4 & A & A & A & A & - & - & - & - & - & - & - & - \\
\hline & 5 & A & A & A & A & - & - & - & - & - & - & - & - \\
\hline & 6 & A & A & A & A & - & - & - & - & - & - & - & - \\
\hline \multirow{6}{*}{$1+2+3+4$} & 1 & A & A & A & A & A & A & A & A & NA & NA & NA & A \\
\hline & 2 & A & A & A & A & NA & A & A & $\mathrm{A}$ & A & NA & A & $\mathrm{A}$ \\
\hline & 3 & A & A & A & A & A & A & A & $\mathrm{A}$ & A & A & A & $\mathrm{A}$ \\
\hline & 4 & A & A & A & A & - & - & - & - & - & - & - & - \\
\hline & 5 & NA & A & A & A & - & - & - & - & - & - & - & - \\
\hline & 6 & A & A & A & A & - & - & - & - & - & - & - & - \\
\hline
\end{tabular}

A= Sequência aleatória; NA= Sequência não aleatória; - =não testado. 
Tabela 1 - Aleatoriedade da produção de fitomassa fresca de vagens para diferentes tamanhos de parcela, por fileira de cultivo, por colheita (C) e agrupamentos de colheitas de feijão-de-vagem (Phaseolus vulgaris L.) cultivado em estufa, túnel e em ambiente não protegido no outono-inverno. Santa Maria, RS, 2010.

\begin{tabular}{|c|c|c|c|c|c|c|c|c|c|c|c|c|c|}
\hline \multirow{3}{*}{$\mathrm{C}$} & \multirow{3}{*}{ Fileiras } & \multicolumn{12}{|c|}{ - } \\
\hline & & 1 & 2 & 3 & 6 & 1 & 2 & 3 & 6 & 1 & 2 & 3 & 6 \\
\hline & & \multicolumn{8}{|c|}{-----------------Estufa----------------- } & \multicolumn{4}{|c|}{-----------Não protegido----------. } \\
\hline \multirow{6}{*}{1} & 1 & A & A & A & A & A & A & A & A & A & A & NA & A \\
\hline & 2 & NA & A & A & A & A & A & A & A & NA & A & A & A \\
\hline & 3 & A & A & A & A & A & A & A & A & A & A & A & A \\
\hline & 4 & A & A & A & A & - & - & - & - & - & - & - & - \\
\hline & 5 & A & A & A & A & - & - & - & - & - & - & - & - \\
\hline & 6 & A & A & A & A & - & - & - & - & - & - & - & - \\
\hline \multirow{6}{*}{2} & 1 & NA & NA & A & A & A & A & A & A & NA & NA & NA & A \\
\hline & 2 & A & A & A & A & A & A & A & A & NA & NA & A & A \\
\hline & 3 & A & A & A & A & A & $\mathrm{A}$ & NA & A & A & A & A & A \\
\hline & 4 & A & A & A & A & - & - & - & - & - & - & - & - \\
\hline & 5 & NA & A & A & A & - & - & - & - & - & - & - & - \\
\hline & 6 & A & A & A & A & - & - & - & - & - & - & - & - \\
\hline \multirow{6}{*}{3} & 1 & A & A & $\mathrm{A}$ & A & A & $\mathrm{A}$ & A & A & NA & NA & NA & A \\
\hline & 2 & A & A & A & A & A & A & A & A & A & A & A & A \\
\hline & 3 & A & A & A & A & A & A & A & A & NA & A & A & A \\
\hline & 4 & A & A & A & A & - & - & - & - & - & - & - & - \\
\hline & 5 & A & A & A & A & - & - & - & - & - & - & - & - \\
\hline & 6 & A & A & A & A & - & - & - & - & - & - & - & - \\
\hline \multirow{6}{*}{4} & 1 & A & A & A & A & A & A & A & A & A & A & A & A \\
\hline & 2 & A & A & A & A & A & A & A & A & A & A & A & A \\
\hline & 3 & NA & A & A & A & A & A & A & A & A & A & A & A \\
\hline & 4 & A & A & A & A & - & - & - & - & - & - & - & - \\
\hline & 5 & A & A & A & A & - & - & - & - & - & - & - & - \\
\hline & 6 & A & A & A & A & - & - & - & - & - & - & - & - \\
\hline \multirow{6}{*}{$1+2$} & 1 & A & A & A & A & A & A & A & A & NA & NA & NA & A \\
\hline & 2 & NA & A & A & A & A & A & A & A & A & A & A & A \\
\hline & 3 & A & A & A & A & A & A & A & A & A & A & A & A \\
\hline & 4 & A & A & A & A & - & - & - & - & - & - & - & - \\
\hline & 5 & A & A & A & A & - & - & - & - & - & - & - & - \\
\hline & 6 & A & A & A & A & - & - & - & - & - & - & - & - \\
\hline \multirow{6}{*}{$3+4$} & 1 & A & A & A & A & A & A & A & A & A & NA & NA & A \\
\hline & 2 & A & A & A & A & A & A & A & A & A & A & A & A \\
\hline & 3 & A & A & A & A & A & A & A & A & A & A & A & A \\
\hline & 4 & A & A & A & A & - & - & - & - & - & - & - & - \\
\hline & 5 & A & A & A & A & - & - & - & - & - & - & - & - \\
\hline & 6 & A & A & A & A & - & - & - & - & - & - & - & - \\
\hline \multirow{6}{*}{$1+2+3+4$} & 1 & A & A & A & A & A & A & A & A & NA & NA & NA & A \\
\hline & 2 & A & A & A & A & NA & A & A & A & A & NA & A & A \\
\hline & 3 & A & A & A & A & A & A & A & A & A & A & A & A \\
\hline & 4 & A & A & A & A & - & - & - & - & - & - & - & - \\
\hline & 5 & NA & A & A & A & - & - & - & - & - & - & - & - \\
\hline & 6 & A & A & A & A & - & - & - & - & - & - & - & - \\
\hline
\end{tabular}


Tabela 3 - Variâncias da fitomassa fresca de vagens $\left(\mathrm{g}^{2} \times 10^{3}\right)$ por fileira de cultivo em cada colheita (C), nos diferentes tamanhos de parcela testados para feijão-de-vagem (Phaseolus vulgaris L.), cultivado em estufa, túnel e em ambiente não protegido, em duas épocas. Santa Maria, RS, 2010.

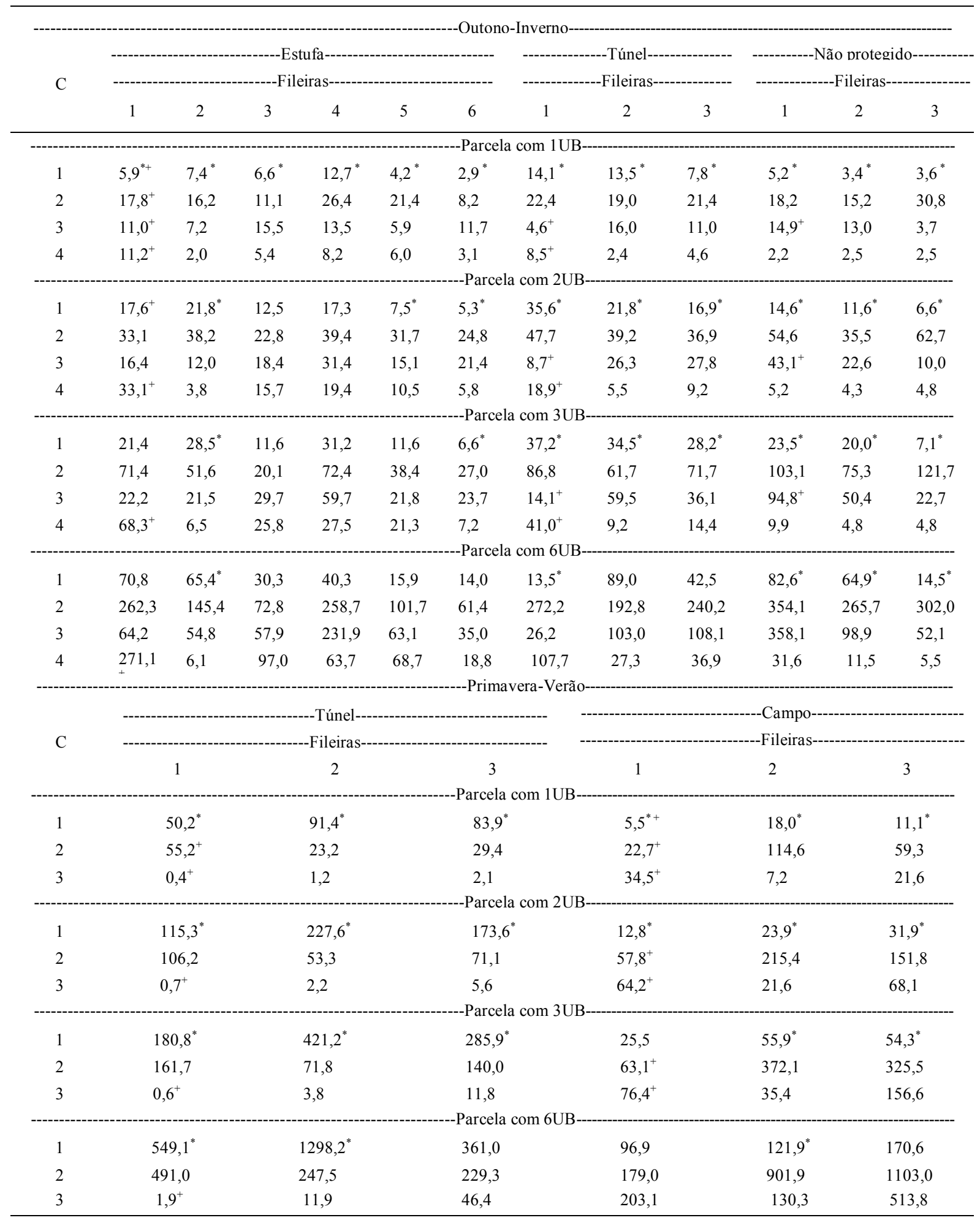

${ }^{*}$ Variâncias heterogêneas entre as colheitas pelo teste de Bartlett em nível de 5\% de probabilidade de erro.

${ }^{+}$Variâncias heterogêneas entre as fileiras de cultivo pelo teste de Bartlett em nível de $5 \%$ de probabilidade de erro.

Ciência Rural, v.42, n.7, jul, 2012. 
estação primavera-verão, o maior número de casos foi no cultivo em ambiente não protegido. Essa diferença entre as épocas, possivelmente ocorreu porque, nos ambientes protegidos, as fileiras laterais estão em uma condição diferenciada de disponibilidade de radiação em relação às centrais, fazendo com que, em condições de baixa disponibilidade de Rg, como no outonoinverno (Figura 1A), ocorra uma maior heterogeneidade entre as linhas. Na primavera-verão, como a Rg não foi um fator limitante (Figura 1C), no ambiente protegido, ocorreu um menor número de casos de heterogeneidade de variâncias entre as fileiras, quando comparado com o ambiente não protegido (Tabela 3 ), confirmando a afirmação anterior. Trabalhando com abóbora italiana, SOUZA et al. (2002) e CARPES et al. (2008) também utilizaram variáveis meteorológicas para explicar as diferenças na variabilidade nos experimentos com a cultura estudada.

No outono-inverno, para a parcela constituida por uma UB, ocorreu heterogeneidade de variâncias entre as fileiras de cultivo em $58 \%$ dos casos e, na primaver/verão, em $83 \%$ (Tabela 3 ). Esses resultados concordam também com os obtidos por CARPES et al. (2008), LÚCIO et al. (2008) e SOUZA et al. (2002), que verificaram heterogeneidade de variâncias entre fileiras em várias colheitas para diversas culturas olerícolas estudadas. Nas duas épocas de cultivo, em todos os experimentos com a parcela constituida por uma UB, foi constatada heterogeneidade de variâncias entre as colheitas em $100 \%$ dos casos (Tabela 3). Esse resultado comprova os obtidos por CARPES et al. (2010), que constataram heterogeneidade de variâncias entre colheitas de abóbora italiana em $100 \%$ dos casos para uma parcela desse mesmo tamanho e ocorreu, dentre outros motivos, porque as vagens de cada colheita se desenvolveram em condições meteorológicas distintas (Figura 1).

De maneira geral, o aumento do tamanho de parcela reduziu os casos de heterogeneidade de variâncias entre as colheitas e entre fileiras. Para o tamanho de parcela constituido por uma UB, a ocorrência de heterogeneidade de variâncias entre colheitas foi de $100 \%$ nas duas épocas de cultivo. Com o uso da parcela constituida por seis UB, os casos reduziram para $42 \%$ no outono-inverno e para $50 \%$ na primavera-verão. A porcentagem de casos de heterogeneidade de variâncias entre as fileiras de cultivos no outono-inverno, que havia sido de de $58 \%$ com o uso de parcelas constituidas por uma UB, reduziu para $8 \%$ para parcelas constituidas por seis UB. Na primavera-verão, ocorreu heterogeneidade de variâncias entre fileiras em $83 \%$ dos casos para parcelas constituidas por uma UB, para a parcela constituida por seis UB a proporção reduziu para $17 \%$ dos casos (Tabela 3). Esses resultados evidenciam que aumentar o tamanho da parcela em experimentos com feijão-devagem é uma estratégia que reduz a heterogeneidade de variâncias entre colheitas e entre fileiras de cultivo.

\section{CONCLUSÃO}

A não aleatoriedade e a variabilidade da produção de fitomassa fresca de vagens são maiores em condições meteorológicas adversas à cultura do feijão-de-vagem e diminuem com o aumento do tamanho de parcela.

O uso de parcelas constituídas por seis unidades básicas (12 plantas), em ambiente protegido ou não protegido, torna a produção de fitomassa fresca de vagens aleatória e proporciona menor variabilidade entre fileiras de cultivo e entre colheitas.

\section{AGRADECIMENTOS}

Ao Conselho Nacional de Desenvolvimento Científico e Tecnológico $(\mathrm{CNPq})$ e à Fundação de Amparo à Pesquisa do Estado do Rio Grande do Sul (FAPERGS), pelo auxílio financeiro para a realização dos experimentos e bolsas de pesquisa e iniciação científica.

\section{REFERÊNCIAS}

ABREU, F.B. et al. Divergência genética entre acessos de feijãode-vagem de hábito de crescimento indeterminado. Horticultura Brasileira, Brasília, v.22, n.3, p.547-552, 2004.

ANDRIOLO, J.L. Fisiologia da produção de hortaliças em ambiente protegido. Horticultura Brasileira, Brasília, v.18, p.26-33, 2000.

BEAVER, R. et al. Statistics for management and economics. 2.ed. North Scituate: Duxbury, 1974. p.366-368.

CARPES, R.H. et al. Ausência de frutos colhidos e suas interferências na variabilidade da fitomassa de frutos de abobrinha italiana cultivada em diferentes sistemas de irrigação. Revista Ceres, Viçosa, v.55, n.6, p.590-595, 2008. Disponível em: $<$ http://www.ceres.ufv.br/CERES/revistas/V55N006P37108.pdf>. Acesso em: 7 dez. 2010.

CARPES R.H. et al. Variabilidade produtiva e agrupamentos de colheitas de abobrinha italiana cultivada em ambiente protegido. Ciência Rural, Santa Maria, v.40, n.2, p.294-301, 2010. Disponível em: <http://www.scielo.br/pdf/cr/v40n2/ a457cr1789.pdf>. Acesso em: 7 dez. 2010. doi: 10.1590/ S0103-84782010005000007.

COUTO, M.R.M. et al. Transformações de dados em experimentos com abobrinha italiana em ambiente protegido. Ciência Rural, Santa Maria, v.39, n.6, p.1701-1707, 2009. Disponível em: <http://www.scielo.br/pdf/cr/v40n2/ a457cr1789.pdf>. Acesso em: 7 dez. 2010. doi: 10.1590/ S0103-84782009005000110. 
EMPRESA BRASILEIRA DE PESQUISA AGROPECUÁRIA EMBRAPA. Centro Nacional de Pesquisa de Solos. Sistema brasileiro de classificação de solos. 2.ed. Rio de Janeiro, 2006. 306p.

FILGUEIRA, F.A.R. Novo manual de olericultura: agrotecnologia moderna na produção de hortaliças. Viçosa: UFV, 2000. p.309-313.

HELDWEIN, A.B. et al. Plastocrono e rendimento de feijãode-vagem cultivado sob ambiente protegido e no ambiente externo em semeadura tardia no outono. Ciência Rural, Santa Maria, v.40, n.4, p.768-773, 2010. Disponível em: $<$ http://www.scielo.br/pdf/cr/v40n4/a522cr2466.pdf $>$. Acesso em: 7 jan. 2011. doi: 10.1590/S0103-84782010005000045.

LOPES, S.J. et al. Técnicas experimentais para tomateiro tipo salada sob estufas plásticas. Ciência Rural, Santa Maria, v.28, n.2, p.193-197, 1998. Disponível em: <http:// www.scielo.br/pdf/cr/v28n2/a02v28n2.pdf $>$. Acesso em: 7 dez. 2010. doi: $10.1590 / \mathrm{S} 0103-84781998000200002$.

LOPES, S.J. et al. Espaçamento entre plantas de sorgo granífero: produtividade de grãos e qualificação do modelo estatístico. Ciência Rural, Santa Maria, v.39, n.3, p.649-656, 2009. Disponível em: <http://www.scielo.br/pdf/cr/v39n3/ a95cr256.pdf >. Acesso em: 7 dez. 2010. doi: 10.1590/S010384782008005000095 .

LÚCIO, A.D. et al. Variação temporal da produção de pimentão influenciada pela posição e características morfológicas das plantas em ambiente protegido. Horticultura Brasileira, Brasília, v.24, n.1, p.31-35, 2006.

LÚCIO, A.D. et al. Variância e média da massa de frutos de abobrinha-italiana em múltiplas colheitas. Horticultura Brasileira, Brasília, v.26, n.3, p.335-341, 2008.
PEREIRA, A.V. et al. Respostas do feijão-vagem cultivado sob proteção com agrotêxtil em duas densidades de plantas. Horticultura Brasileira, Brasília, v.21, n.3, p.564-569, 2003.

PRELA, A.; RIBEIRO, A.M.A. Determinação de graus-dia acumulados e sua aplicação no planejamento do cultivo de feijão vagem (Phaseolus vulgaris L.) para Londrina-PR. Revista Brasileira de Agrometeorologia, Santa Maria, v.10, n.1, p.83-86, 2002.

SANTOS, D. et al. Suficiência amostral para alface cultivada em diferentes ambientes. Ciência Rural, Santa Maria, v.40, n.4, p.800-805, 2010. Disponível em: <http://www.scielo.br/ $\mathrm{pdf} / \mathrm{cr} / \mathrm{v} 40 \mathrm{n} 4 / \mathrm{a} 554 \mathrm{cr} 1846 . \mathrm{pdf}>$. Acesso em: $7 \mathrm{dez}$. 2010. doi: 10.1590/S0103-84782010000400009.

SOUZA, M.F. et al. Tamanho da amostra para peso da massa de frutos, na cultura da abóbora italiana em estufa plástica. Revista Brasileira de Agrociência, Pelotas, v.8, n.2, p.131-136, 2002. Disponível em: <http://www.ufpel.tche.br/faem/agrociencia/ v8n2/artigo07.pdf $>$. Acesso em: 7 dez. 2010.

STEEL, R.G.D. et al. Principles and procedures of statistics abiometrical approach. 3.ed. Nova York: McGraw-Hill, 1997. 666p.

STORCK, L. et al. Experimentação vegetal. 2.ed. Santa Maria: UFSM, 2006. 198p.

ZANARDO, B. et al. Posições das mudas de alface nas bandejas de poliestireno e efeitos na normalidade e homogeneidade dos erros na produção de plantas. Revista Ciência Agronômica, Fortaleza, v.41, n.2, p.285-293, 2010. Disponível em: <http:/ /www.scielo.br/pdf/rca/v41n2/v41n2a17.pdf >. Acesso em: 7 dez. 2010. doi: 10.1590/S1806-66902010000200017. 